\title{
The field-dependence of the solid-state photo-CIDNP effect in two states of heliobacterial reaction centers
}

\author{
Smitha Surendran Thamarath $\cdot$ A. Alia • \\ Esha Roy $\cdot$ Karthick Babu Sai Sankar Gupta • \\ John H. Golbeck · Jörg Matysik
}

Received: 31 January 2013/Accepted: 14 May 2013/Published online: 31 May 2013

(C) Springer Science+Business Media Dordrecht 2013

\begin{abstract}
The solid-state photo-CIDNP (photochemically induced dynamic nuclear polarization) effect is studied in photosynthetic reaction centers of Heliobacillus mobilis at different magnetic fields by ${ }^{13} \mathrm{C}$ MAS (magic-angle spinning) NMR spectroscopy. Two active states of heliobacterial reaction centers are probed: an anaerobic preparation of heliochromatophores ("Braunstoff", German for "brown substance") as well as a preparation of cells after exposure to oxygen ("Grünstoff", "green substance"). Braunstoff shows significant increase of enhanced absorptive (positive) signals toward lower magnetic fields, which is interpreted in terms of an enhanced differential relaxation (DR) mechanism. In Grünstoff, the signals remain emissive (negative) at two fields, confirming that the influence of the DR mechanism is comparably low.
\end{abstract}

Keywords Heliobacteria - Electron transfer .

Photo-CIDNP · Solid-state NMR · Magnetic field effect

\section{Abbreviations}

$\begin{array}{ll}\text { ALA } & \text { Aminolevulinic acid } \\ \text { BChl } & \text { Bacteriochlorophyll }\end{array}$

S. Surendran Thamarath · A. Alia - E. Roy .

K. B. Sai Sankar Gupta · J. Matysik

Leiden Institute of Chemistry, Leiden University,

Einsteinweg 55, 2300 RA Leiden, The Netherlands

S. Surendran Thamarath $\cdot$ A. Alia $\cdot$ J. Matysik $(\bowtie)$

Institut für Analytische Chemie, Universität Leipzig, Linnéstr. 3, 04103 Leipzig, Germany

e-mail: joerg.matysik@uni-leipzig.de

J. H. Golbeck

Department of Chemistry, Department of Biochemistry and Molecular Biology, The Pennsylvania State University,

328 South Frear Laboratory, University Park, PA 16802, USA

$\begin{array}{ll}\text { BPhe } & \text { Bacteriopheophytin } \\ \text { Chl } & \text { Chlorophyll } \\ \text { Chl } a_{F} & \text { Chlorophyll } a \text { with a farnesyl side chain } \\ \text { CI } & \text { Continuous illumination } \\ \text { DD } & \text { Differential decay } \\ \text { DR } & \text { Differential relaxation } \\ \text { EPR } & \text { Electron paramagnetic resonance } \\ \text { FT-IR } & \text { Fourier transform infrared spectroscopy } \\ \text { Hb. } & \text { Heliobacillus } \\ \text { ISC } & \text { Intersystem crossing } \\ \text { MAS } & \text { Magic angle spinning } \\ \text { NMR } & \text { Nuclear magnetic resonance } \\ \text { P } & \text { Special pair primary electron donor } \\ \text { Photo-CIDNP } & \text { Photo-chemically induced dynamic } \\ & \text { nuclear polarization } \\ \text { PS I } & \text { Photosystem I } \\ \text { PS II } & \text { Photosystem II } \\ R b . & \text { Rhodobacter } \\ \text { RC } & \text { Reaction center } \\ \text { RPM } & \text { Radical pair mechanism } \\ S & \text { Singlet } \\ T_{0} & \text { Triplet } \\ \text { TPPM } & \text { Two-pulse phase-modulation } \\ \text { TR } & \text { Time resolved } \\ \text { TSM } & \text { Electron-electron nuclear three spin } \\ \text { WT } & \text { mixing } \\ & \text { Wild type } \\ \end{array}$

\section{Introduction}

Heliobacillus (Hb.) mobilis is a strictly anaerobic, nitrogen fixing photosynthetic organism found in rice paddy fields (Gest 1994; Stevenson et al. 1997; Golbeck 2007). The photosynthetic reaction center (RC) of Hb. mobilis consists 
of a symmetric polypeptide homodimer belonging to the Type-I RC family, as defined by the presence of an interpolypeptide iron-sulfur cluster, $F_{\mathrm{X}}$, as an electron acceptor (Vassiliev et al. 2001). Thus, there is no structural information available for any homodimeric Type I RC. The absorption spectrum of anaerobically grown $\mathrm{Hb}$. mobilis cells is shown in Fig. 1 (red spectrum). The color appears brownish green and is therefore called "Braunstoff". A unique pigment, bacteriochlorophyll $g$ (BChl $g$, see Fig. 2 left), has been identified in these bacteria (Brockmann and Lipinski 1983). BChlg acts as an antenna pigment, and two 13 (Stevenson et al. 1997) epimers $\left(\mathrm{BChl} g^{\prime}\right)$ act as the symmetric primary electron donor special pair $\left(\mathrm{P} 798^{+}\right)$ (Kobayashi et al. 1991; Surendran Thamarath et al. 2012a). The primary electron acceptor is $8^{1}$-hydroxy chlorophyll $a$ ( $8^{1}$-OH Chl $a_{F}$; Fig. 2, right) (Vandemeent et al. 1991). The intense absorbance peaks at 570 and $798 \mathrm{~nm}$ correspond to BChlg. The less intense peak at $670 \mathrm{~nm}$ corresponds to $8^{1}-\mathrm{OH} \mathrm{Chl} a_{F}$, where $F$ refers to the farnesyl side chain (Vandemeent et al. 1991). Both isolated RCs and membranes have been studied by FT-IR (Nabedryk et al. 1996), EPR techniques (Prince et al. 1985), and photoCIDNP MAS NMR (Surendran Thamarath et al. 2012a; Roy et al. 2008a).

A peculiarity of BChlg is the photoconversion at pyrrole ring II when exposed to oxygen, a process that is accelerated in the presence of light. The color change from brownish green to emerald green is observed during this process and leads to a product called Grünstoff. The pigment becomes spectroscopically equivalent to Chl $a$, which is present in cyanobacteria and green plants. The absorption spectrum of $\mathrm{Hb}$. mobilis after this process is shown at Fig. 1 (green spectrum). The peak at $670 \mathrm{~nm}$, indicating

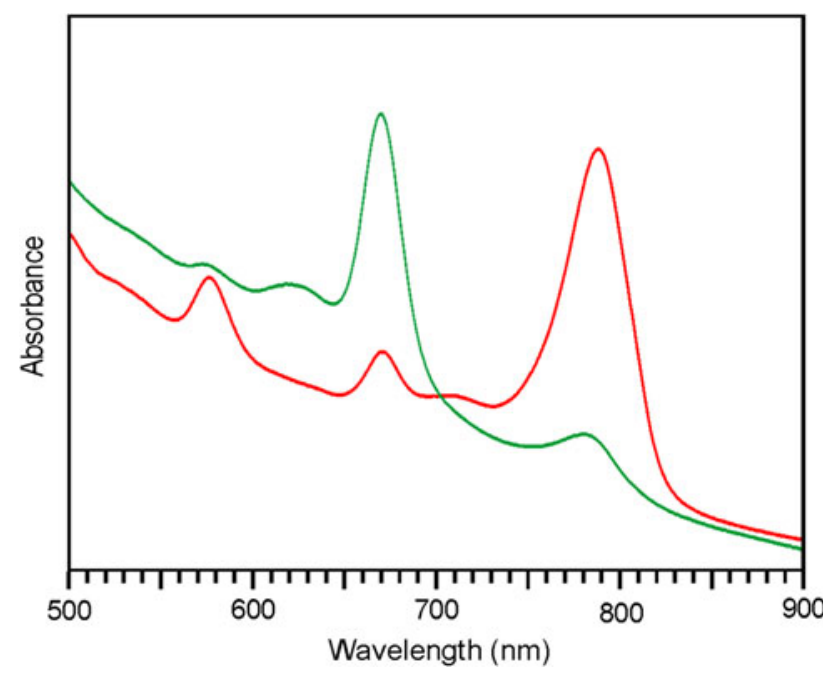

Fig. 1 Absorption spectra of heliobacterial cells in the anaerobic (Braunstoff, red) and aerobic (Grünstoff, green) states. The absorbance is presented in arbitrary units
Chl $a_{F}$, becomes more intense. All the absorbance peaks of the BChlg pigment decrease significantly. The small remaining peak at $798 \mathrm{~nm}$ may be due to the special pair which remains as $\mathrm{BChlg}^{\prime}$ as shown by photo-CIDNP MAS NMR directly on whole cells (Surendran Thamarath et al. 2012a). In that study, it was assumed that the color change by phototransformation affects, in addition to the antenna pigments, also the accessory cofactors. That conversion modifies electron-electron interactions of the radical pair leading to different contributions of the mechanisms that cause the solid-state photo-CIDNP effect.

The solid-state photo-CIDNP effect, discovered in 1994 by Zysmilich and McDermott (1994), has been observed in all naturally occurring RCs studied to-date, and it has been assumed that the effect is an intrinsic property of lightinduced electron transfer in photosynthesis (Matysik et al. 2009). In this effect, the non-Boltzmann nuclear spin polarization is detected as strongly enhanced ${ }^{13} \mathrm{C}$ or ${ }^{15} \mathrm{~N}$ MAS NMR signal (Daviso et al. 2008). The non-Boltzmann spin distribution is achieved by the transfer of the initially high-electron spin order from the primary radical pair to the nuclear spins by hyperfine interaction (Jeschke and Matysik 2003). Such transfer at the high-magnetic fields as applied in an NMR experiment is explained by one or more three parallel mechanisms that occur in the solid state under conditions of continuous illumination: (Prakash et al. 2005; Prakash et al. 2006; Daviso et al. 2009) three spin mixing (TSM) (Jeschke 1997), differential decay (DD) (Polenova and McDermott 1999), and differential relaxation (DR) (McDermott et al. 1998). These mechanisms are well understood in photo-CIDNP MAS NMR studies of the quinone-removed RCs of $R b$. sphaeroides and in theoretical simulations based on these experiments (Jeschke and Matysik 2003; Daviso et al. 2009). Scheme 1 shows the spin-chemical cycle process that lead to high-nuclear polarization in RCs of $R b$. sphaeroides WT and the carotenoid-less mutant R26. Under illumination with white light, the primary electron donor, which is a special pair of bacteriochlorophyll $a(\mathrm{BChl} a)$ molecules, is excited and forms a radical cation by donating an electron to the primary electron acceptor $\Phi$, a bacteriopheophytin (BPhe). This leads to the formation of a spin-correlated radical pair, initially in its singlet state $(S)$, which converts to the triplet state $\left(T_{0}\right)$ by intersystem crossing (ISC). The high-electron spin order of the initial pure electronic singlet state is transferred to a net nuclear polarization by two parallel mechanisms, the TSM (Jeschke 1997) and the DD mechanisms (Polenova and McDermott 1999).

The TSM mechanism is explained by the combined action of electron-electron dipolar coupling or exchange coupling and the pseudosecular hyperfine coupling (HFC) $B=\left(A_{\mathrm{zx}}^{2}+A_{\mathrm{zy}}^{2}\right)^{1 / 2}$, which break the antisymmetry of the nuclear spin population in a coherent spin evolution of the 
Fig. 2 Chemical structure of BChlg (left) and $8^{1}-\mathrm{OH}$ BChla (right)

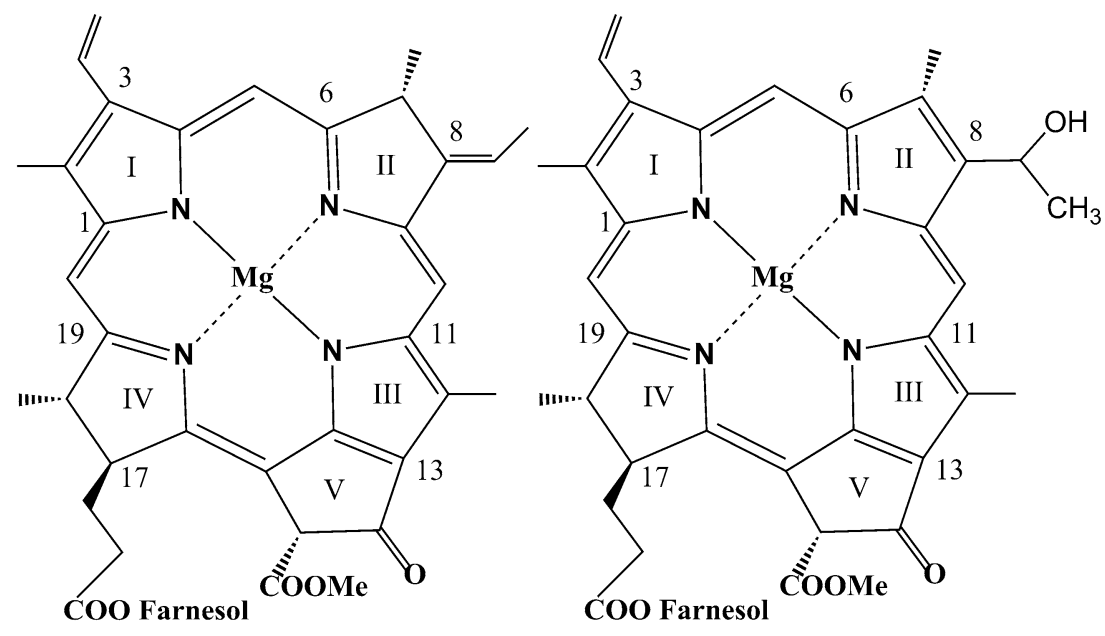

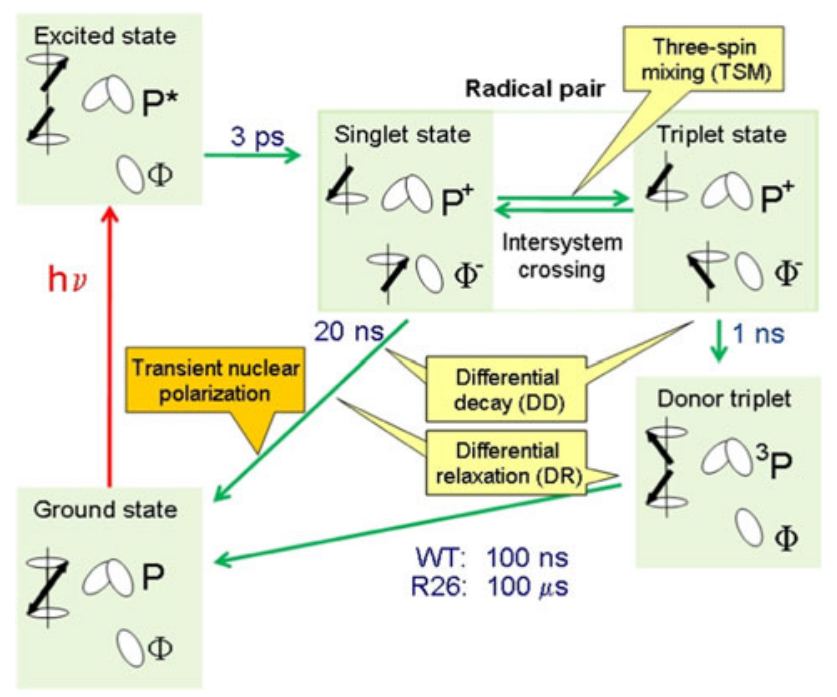

Scheme 1 Photocycle in quinone-blocked RCs of $R b$. sphaeroides WT and R26. Upon illumination and fast electron transfer from an excited singlet state, a radical pair is formed in a pure singlet state having a high-electron spin order. The radical pair is formed by a radical cation at the two donor $\mathrm{BChls} \mathrm{(special} \mathrm{pair,} P$ ) and a radical anion on the BPhe acceptor cofactor $(\Phi)$ of the active branch. The chemical fate of the radical pair depends on its electronic spin state: while the singlet state is allowed to recombine, for the triplet state a direct recombination is spin-forbidden and a donor triplet $\left({ }^{3} \mathrm{P}\right)$ is formed by inter-system crossing. Mechanisms that build up photoCIDNP under steady-state conditions are labeled in yellow. Transient nuclear polarization, observable in time-resolved experiments, is labeled in orange

spin correlated radical pair (Jeschke 1997). Due to the different lifetimes of the $S$ and of the $T_{0}$ states, antisymmetry of the nuclear spin population in the spin correlated radical pair can be broken by a buildup of net nuclear polarization via the pseudosecular HFC $B$ in a DD mechanism. The polarization transfer by the DD mechanism occurs due to a single matching condition of $2\left|\omega_{\mathrm{I}}\right|=\left|A_{\mathrm{zz}}\right|$, and the difference of singlet and triplet radical pair lifetimes must be of the order of the inverse hyperfine splitting, which is defined as $\left.\left[\left(\omega_{\mathrm{I}}+A / 2\right)^{2}+B^{2} / 4\right)\right]^{1 / 2}-$ $\left.\left[\left(\omega_{\mathrm{I}}-A / 2\right)^{2}+B^{2} / 4\right)\right]^{1 / 2}$. The electron Zeeman interaction drives nuclear-spin independent interconversion between the singlet and triplet state of the radical pair, thus not affecting nuclear spin populations. This remains true even in the presence of $B$, since this coupling is too small to mix different electron spin states. Only if electron spin states are mixed by electron-electron coupling $(d)$, as in the TSM mechanism, mixing of nuclear spin states by $B$ will also be affected by an electron spin state mixing. If the singlet and triplet state of the radical pair have different lifetimes, as in the DD mechanism, pairs in these two states will experience hyperfine evolution for a different time, which also breaks the antisymmetry of the nuclear spin populations. In the absence of $B$, the contribution of the nuclear Zeeman interaction $\left(\omega_{\mathrm{I}}\right)$ to spin evolution is independent of the electron spin state and will thus not lead to any polarization transfer. In the presence of such coupling, the ratio between the magnitude of $\omega_{\mathrm{I}}$ and $A_{\mathrm{zz}}$ determines the extent of mixing of nuclear spin states, and thus also the extent to which population antisymmetry is violated. The emissive signals of the photo-CIDNP MAS NMR spectrum of WT RCs are due to the predominance of the TSM mechanism over the DD mechanism, and the contribution of the DD to emissive/absorptive patterns depends on the $\Delta g$ value of electron spins which is negative for bacteriochlorophyll (Jeschke and Matysik 2003). The relative intensity of these emissive photo-CIDNP MAS NMR signals provides information about the spin density distribution of the radical pair in RCs (Prakash et al. 2005).

In the carotenoid-less mutant R26 of Rb. sphaeroides RCs that have a long lived donor triplet transfer polarization by an additional DR mechanism (Prakash et al. 2006; McDermott et al. 1998; Surendran Thamarath et al. 2012a). This is a modified radical pair mechanism (RPM) and depends on the different nuclear longitudinal relaxation rates (Jeschke and Matysik 2003; Goldstein and Boxer 
1987; Closs 1975). While electron polarization does not build up during subsequent photocycles, the long ${ }^{13} \mathrm{C} T_{1}$ time (Daviso et al. 2008) allows for the accumulation of nuclear polarization in a photo-CIDNP MAS NMR experiment. The effect has been shown to allow for a signal enhancement of greater than 80,000, and hence for the detection of cofactors directly in whole cells (Surendran Thamarath et al. 2012a; Prakash et al. 2006; Janssen et al. 2010). Recently, it has been demonstrated that the effect is not limited to frozen natural photosynthetic RCs at NMR fields, but it also occurs in a blue-light photoreceptor (Surendran Thamarath et al. 2010), in liquid membranes (Daviso et al. 2011), and is predicted to occur at earth's magnetic field strength (Jeschke et al. 2011). Since the effect relies on hyperfine interactions, it requires a radical pair lifetime of at least some tens of nanoseconds. Hence, any observation of the effect demonstrates that lightinduced radical pairs having a lifetime significantly longer than ten nanoseconds are present in a sample.

The mechanisms that cause the solid-state photo-CIDNP effect show different field dependencies for signal enhancement. Such studies have contributed to the discussion on the mechanisms (Jeschke and Matysik 2003; Prakash et al. 2005; Prakash et al. 2006), and might allow for disentangling the different contributions (Surendran Thamarath et al. 2012a). In addition, since short radicalpair lifetimes lead to a broadening of the polarization-field curves (Closs 1977), the bandwidths of the enhancement curve can be taken as indication of the length of the radical pair lifetime. Field-dependent studies have been done for RCs of Rb. sphaeroides (Prakash et al. 2005; Prakash et al. 2006; Surendran Thamarath et al. 2012a) Photosystems I and II (Roy et al. 2007), green sulfur bacteria (Roy et al. 2008b), and-for a smaller field range_on Braunstoff (Roy et al. 2008a). Here, we re-investigate the magnetic field dependence of Braunstoff in heliobacteria in a larger field range and include Grünstoff in the study.

\section{Materials and methods}

Sample preparation

Cells of Hb. mobilis strain ATCC 43427 (DSMZ 6151) were used in this study. The cells were cultured in medium no. 1552 (van de Meent et al. 1990) anaerobically at $37{ }^{\circ} \mathrm{C}$ under continuous light. After 7 days of growth, cells were harvested by centrifugation $(4,000 \mathrm{rpm})$. One half of the harvested cells were uniformly suspended in deoxygenated $20 \mathrm{mM}$ TrisHCl buffer ( $\mathrm{pH} 8.0$ ) containing $10 \mathrm{mM}$ sodium ascorbate. Under nitrogen gas flow, this sample (Braunstoff) was reduced with $50 \mathrm{mM}$ sodium dithionite and packed in a 4-mm sapphire rotor for MAS NMR experiments. The other half of the harvested cells was used for preparing Grünstoff. These cells were washed with the medium no. 1552 that does not contain sodium ascorbate. The cells were re-suspended in the same nonascorbate medium, and were bubbled with oxygen gas under illumination. The conversion to Grünstoff was monitored by taking absorption spectra every $15 \mathrm{~min}$. After $3 \mathrm{~h}$, the conversion to Grünstoff was almost complete and the cells were collected by centrifugation. The cells were resuspended in $20 \mathrm{mM}$ TrisHCl buffer ( $\mathrm{pH}$ 8) containing $10 \mathrm{mM}$ sodium ascorbate, and reduced with $50 \mathrm{mM}$ sodium dithionite under nitrogen gas flow. This sample (Grünstoff) was packed in a $4 \mathrm{~mm}$ sapphire rotor for MAS NMR experiments.

The membrane fragments (Heliochromatophores) were prepared (Roy et al. 2008a) by sonication of the cells for $35 \mathrm{~min}$ followed by a $15 \mathrm{~min}$ centrifugation at $40,000 \times \mathrm{g}$. The resulting supernatant was ultracentrifuged for $2 \mathrm{~h}$ at $200,000 \times g$ at a temperature of $4^{\circ} \mathrm{C}$. The pellet containing the membrane fragments was resuspended in $50 \mathrm{mM}$ glycine buffer with $0.02 \%$ sulfobetaine-12 (SB-12) detergent $(\mathrm{pH}$ 10.8). The sample was reduced by $50 \mathrm{mM}$ sodium dithionite under nitrogen gas flow and packed in a $4 \mathrm{~mm}$ sapphire rotor for photo-CIDNP MAS NMR experiments.

\section{MAS-NMR Measurements}

All ${ }^{13} \mathrm{C}$ and ${ }^{15} \mathrm{~N}$ MAS NMR experiments of $H b$. mobilis cells were measured in wide-bore NMR spectrometers operating at 17.6, 9.4, 4.7, and 2.4 T equipped with 4-mm MAS probes (Bruker, Karlsruhe, Germany). The sample was packed into an optically transparent 4-mm MAS sapphire rotor and inserted into the MAS probe. A very lowspinning frequency of $500 \mathrm{~Hz}$ was applied during freezing to achieve a homogeneous distribution of sample against the rotor wall (Fischer et al. 1992). The spinning frequency was increased to $8 \mathrm{kHz}$ after the sample was completely frozen at $235 \mathrm{~K}$. For continuous illumination photo-CIDNP MAS NMR experiments, white light from a $1000 \mathrm{~W}$ Xenon lamp was used. Both dark and photo-CIDNP spectra were obtained with a simple Hahn-echo pulse sequence (Matysik et al. 2000; Matysik et al. 2001) with TPPM (two-pulse phase modulation) proton decoupling (Bennett et al. 1995).

In all NMR experiments, a recycle delay of $2 \mathrm{~s}$ was applied. ${ }^{13} \mathrm{C}$ MAS NMR signals of natural abundant samples were collected for about 2 days. Both dark and light ${ }^{13} \mathrm{C}$ MAS NMR spectra were referenced to the ${ }^{13} \mathrm{COOH}$ chemical shift of solid tyrosine. $\mathrm{HCl}$ at $172.1 \mathrm{ppm}$, and artificial line broadening of $50 \mathrm{~Hz}$ was applied prior to Fourier transformation. For illumination, a $1000 \mathrm{~W}$ xenon arc lamp producing continuous white light was used and the radiation was transferred into the NMR probe via a fiber bundle (Matysik et al. 2000). 


\section{Results and discussions}

Photo-CIDNP ${ }^{13} \mathrm{C}$ MAS NMR on Braunstoff at different magnetic fields

In Fig. $3,{ }^{13} \mathrm{C}$ MAS NMR spectra of natural abundance heliochromatophores of Braunstoff at four magnetic field strengths are shown without illumination of the sample. The magnetic fields are $17.6 \mathrm{~T}\left(750 \mathrm{MHz}{ }^{1} \mathrm{H}\right.$ frequency, spectrum A), $9.4 \mathrm{~T}(400 \mathrm{MHz}, \mathrm{B}), 4.7 \mathrm{~T}(200 \mathrm{MHz}, \mathrm{C})$, and $2.4 \mathrm{~T}(100 \mathrm{MHz}, \mathrm{D})$. There is, as expected for NMR spectroscopy on Boltzmann populations, a clear tendency for an improved signal to noise ratio toward higher fields. Figure 4 shows the complementary photo-CIDNP ${ }^{13} \mathrm{C}$ MAS NMR spectra obtained from natural abundance heliochromatophores of Braunstoff under continuous illumination with white light at magnetic fields of 17.6 (A), 9.4 (B), 4.7 (C), and 2.4 T (D). The signal enhancement is significant at all the fields and is especially strong at the lower fields. Comparison of Figs. 3 and 4 demonstrates occurrence and spectral features of the solid-state photo-CIDNP effect and its field dependence. Figure 5 provides a zoom into the region of light-induced signals. Toward lower fields, the chemical shift dispersion decays, but the intensity enhancement increases. Therefore, the highest spectral

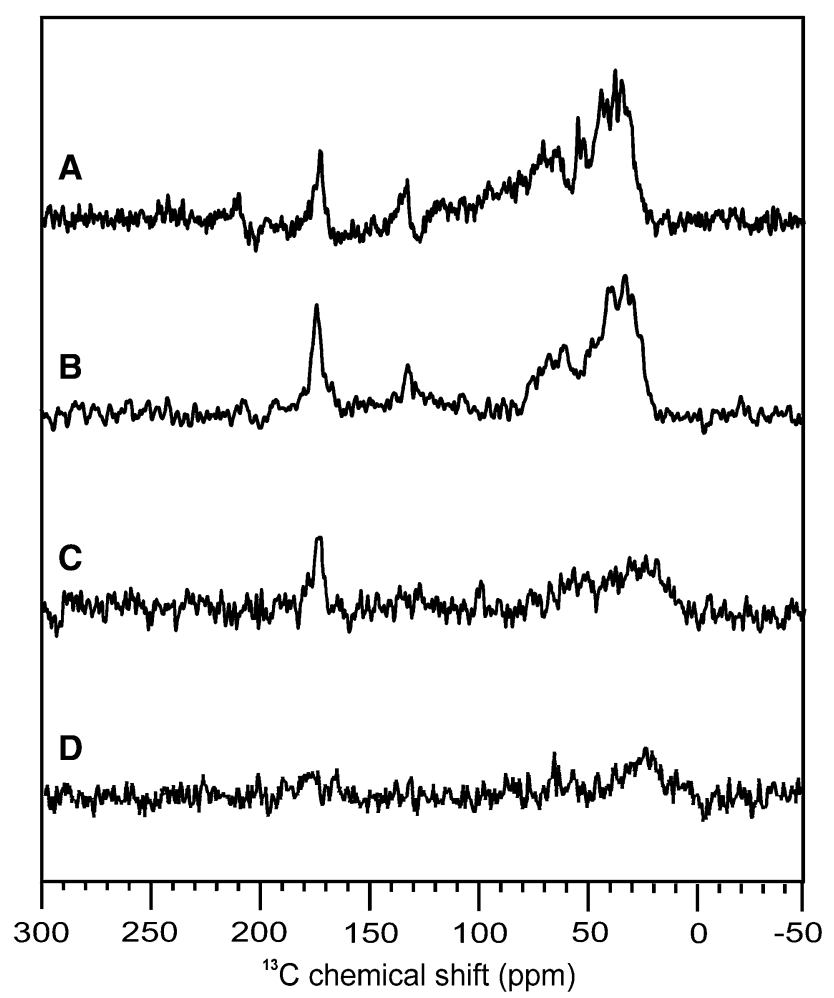

Fig. $3{ }^{13} \mathrm{C}$ MAS NMR spectra of Braunstoff at $A$ 17.6, B 9.4, C 4.7 and $D 2.4 \mathrm{~T}$. Spectra are obtained from heliochromatophores in the dark

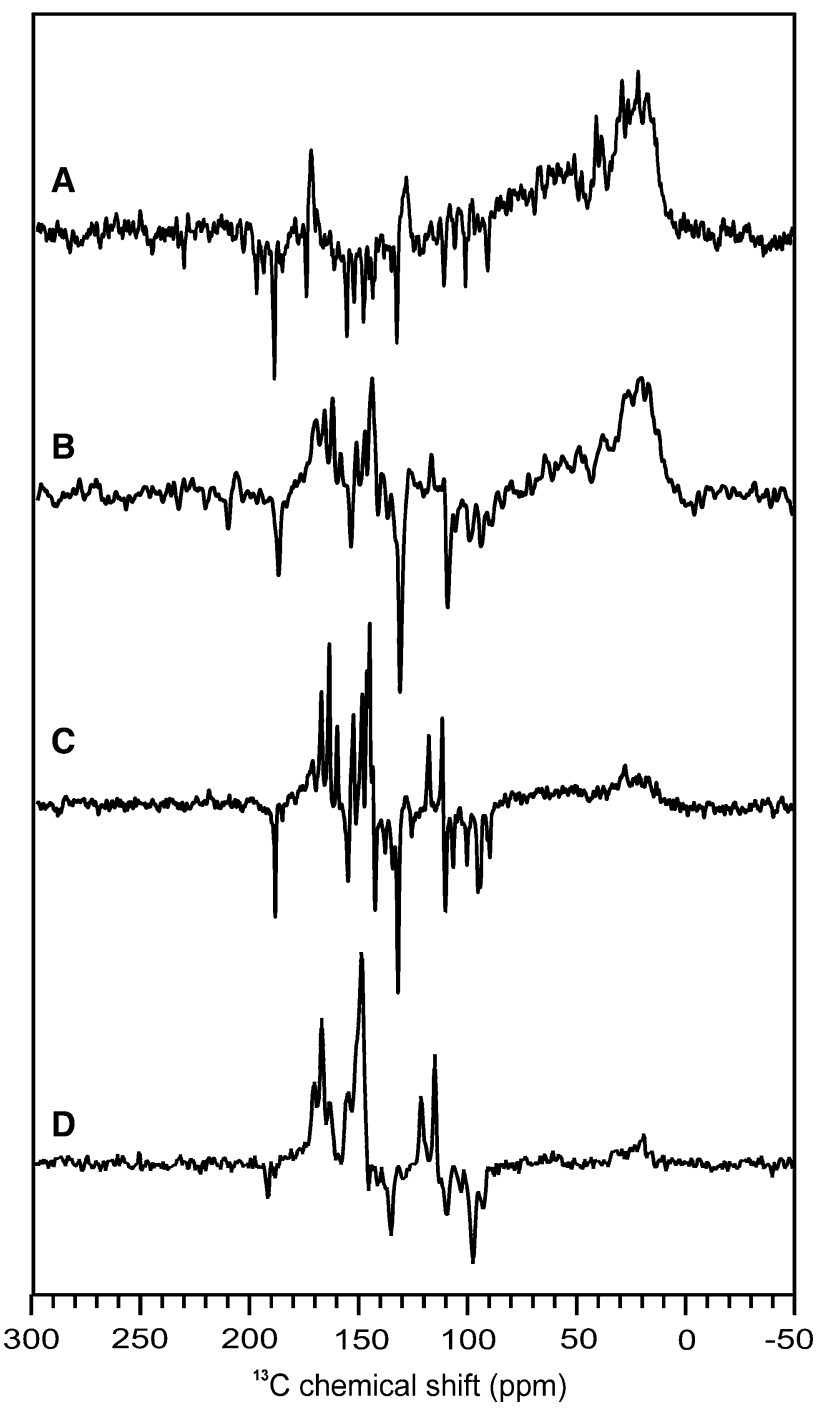

Fig. $4{ }^{13} \mathrm{C}$ photo-CIDNP MAS NMR spectra of Braunstoff at $A$ 17.6, $B$ 9.4, $C 4.7$ and $D 2.4 \mathrm{~T}$. Spectra are obtained from heliochromatophores under illumination with continuous white light

quality is obtained at $4.7 \mathrm{~T}$ (Spectrum C). The frequencies remain constant upon field change. Tentative assignments (Table 1) have been obtained in previous study (Surendran Thamarath et al. 2012a). This work shows that the positive signals originate from the symmetric $\mathrm{BChlg}{ }^{\prime}$ donor special pair, and the negative signals from the $8^{1}-\mathrm{OH}$ Chl $a_{F}$ acceptors of both branches.

Positive light-induced signals are labeled in red and negative light-induced signals are labeled in green. While at 17.6 $\mathrm{T}$ all light-induced signals are emissive (negative), at lower fields enhanced absorptive (positive) signals arise and become dominant. At $2.4 \mathrm{~T}$, almost all the features are enhanced absorptive. The data show that the intensity ratio of the positive signals remains rather constant upon field change. Interestingly, the negative signals show individual field dependencies. At high field (Fig. 5, Spectrum A), a set of 


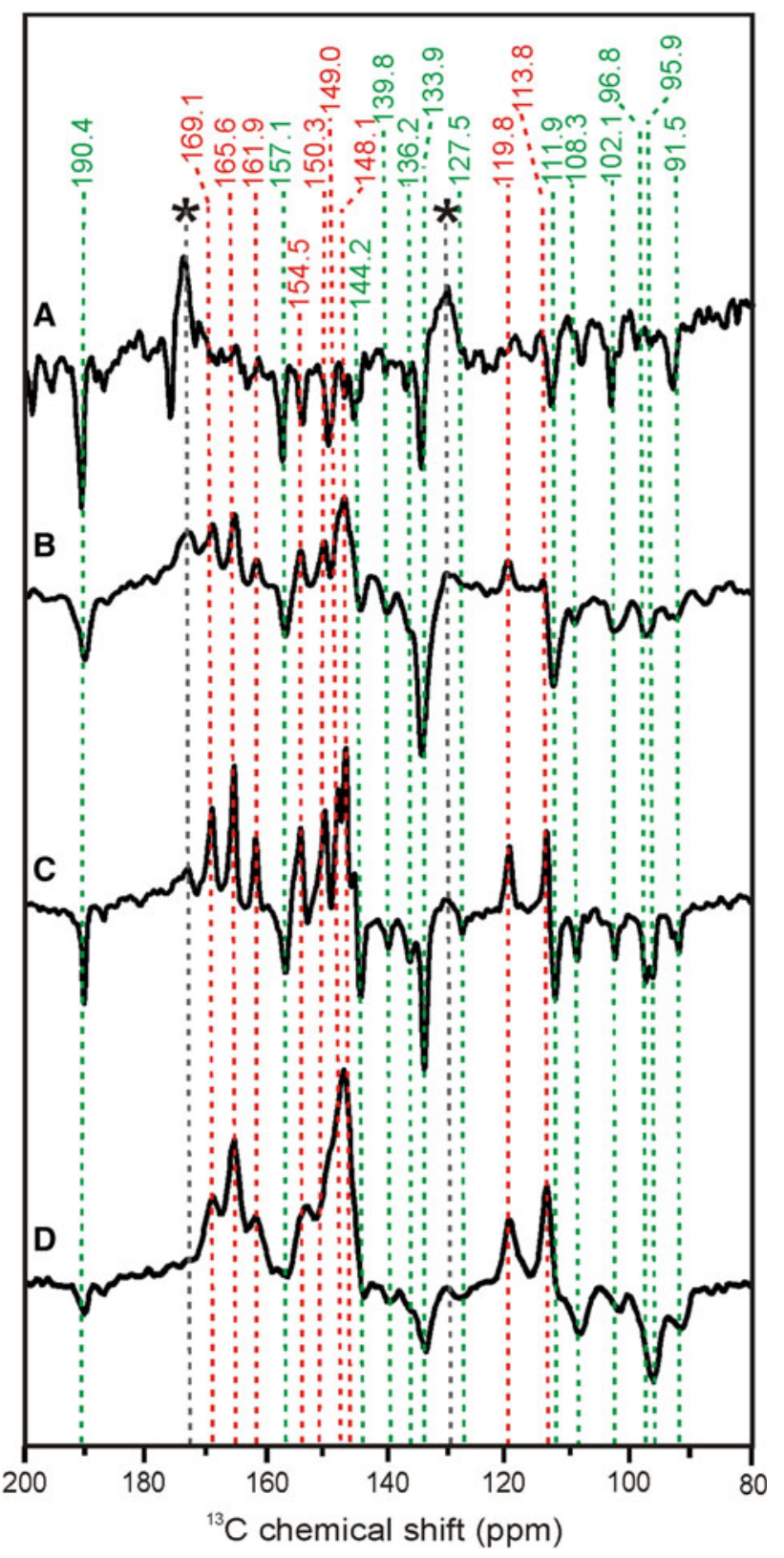

Fig. 5 Selected region of the ${ }^{13} \mathrm{C}$ photo-CIDNP MAS NMR spectra of Braunstoff at $A$ 17.6, $B$ 9.4, $C 4.7$ and $D 2.4$ T. Spectra are obtained from heliochromatophores under illumination with continuous white light

about a dozen of signals of rather similar intensity is observed, from which only a few remain strong at lower fields (Spectrum D). These strong signals are at 190.4, 133.9, 111.9, and a slightly split doublet at $\sim 96 \mathrm{ppm}$ and assigned to the acceptor carbons $\mathrm{C}-8^{1}, \mathrm{C}-3, \mathrm{C}-3^{2}$, and C-5, respectively.

\section{Photo-CIDNP ${ }^{13} \mathrm{C}$ MAS NMR on Grünstoff at different magnetic fields}

In Figs. 6 and $7,{ }^{13} \mathrm{C}$ MAS NMR spectra of Grünstoff obtained in the dark and under illumination at fields of 4.7
(A) and $2.4 \mathrm{~T}$ (B) are shown. Comparison shows the occurrence of the solid-state photo-CIDNP effect as indicated by the light-induced emissive signals. Also in the case of Grünstoff, the field at $4.7 \mathrm{~T}$ provides an excellent compromise for both enhancement and spectral dispersion. Except for some weak positive features, both spectra are emissive.

To compare spectra of both the sample states at this field (Fig. 8), Braunstoff is shown measured in whole cells (Spectum 8A) which leads to a slight broadening of the signals compared to the heliochromatophore preparation at the same field (Spectrum 5C). Comparing spectra of Braunstoff (Spectrum 8A) and Grünstoff (Spectrum 8B), it is evident that the chemical shifts are not affected by the transformation but the intensity pattern is strongly changed. The constancy of the chemical shifts was interpreted in terms of identical and chemically unmodified donor and acceptor cofactors (Surendran Thamarath et al. 2012a). The change of the signal intensity patterns was interpreted as a change in the ratio of the contributions of different enhancement mechanisms. It was assumed that in addition to the antenna cofactors, the accessory cofactors were modified. This interpretation was supported by a more mobile molecular triplet state in Grünstoff. A change in the enhancement mechanism would affect the interactions within the radical pair and therefore the relative contributions of TSM, DD, and DR. The weakness of the positive signals in Grünstoff has been interpreted as a lowering of the DR contribution or as an increase of the TSM. It is remarkable that three of the signals which become stronger emissive in Braunstoff (90.3, 133.9, and $111.9 \mathrm{ppm})$ become less emissive in Grünstoff.

\section{Contributions of different mechanisms}

Since the kinetics of cyclic electron transfer and triplet dynamics are similar to RCs of $R b$. sphaeroides $\mathrm{R} 26$ (Surendran Thamarath et al. 2012a), in Braunstoff TSM and DR are expected to decay at lower fields. The strong increase of positive signal intensity is certainly due to DR. Now one might speculate also that the negative signals at $2.4 \mathrm{~T}$ are caused by DR. In fact, the carbon signals showing strongly emissive signals are those acceptor carbons which are oriented toward the donor, the site of the molecular triplet. On the other hand, occurrence of the DR at the acceptor would be remarkable since the relaxation depends on the square of the anisotropy of the hyperfine tensor. While there are large HFCs at the donor, due to electron spin densities directly at the carbons, at the acceptor interaction would need to be through space, and even if it would be ten times weaker, the relaxation effect would be 100 times weaker. The alternative would be to explain the modification of the intensity pattern of negative signals by an alteration of the competing effects of TSM (emissive) 
Table 1 Tentative chemical shift assignments for signals observed by ${ }^{13} \mathrm{C}$ photo-CIDNP MAS NMR from whole cells of $\mathrm{Hb}$. mobilis in its anoxygenic (Braunstoff) and oxygenic (Grünstoff) states

\begin{tabular}{|c|c|c|}
\hline Carbon no. & Hb. mobilis (Braunstoff) & Hb. mobilis (Grünstoff) \\
\hline $13^{1}$ & 190.3(E) & 190.1(E) \\
\hline $17^{3}$ & - & - \\
\hline $13^{3}$ & - & - \\
\hline 19 & $165.6(\mathrm{~A})$ & $165.6(\mathrm{~A})$ \\
\hline 14 & 161.1(A) & - \\
\hline 1 & $154.5(\mathrm{~A})$ & 154.5(A), 156.7(E) \\
\hline 6 & $169.1(\mathrm{~A})$ & 169.1(A), 171.5(E) \\
\hline 16 & $150.3(\mathrm{~A})$ & 153.2(E) \\
\hline 4 & 148.1(A) & 153.2(E) \\
\hline 11 & $145.8(\mathrm{~A})$ & $145.8(\mathrm{~A})$ \\
\hline 9 & $150.2(\mathrm{~A})$ & $149.0(\mathrm{E})$ \\
\hline 8 & - & 144.7(E) \\
\hline $8^{1}$ & $64.3(\mathrm{E})$ & $64.3(\mathrm{E})$ \\
\hline 3 & - & 139.8(E) \\
\hline 2 & 133.9(E) & 133.9(E) \\
\hline 12 & $119.8(\mathrm{~A})$ & 119.8(A) \\
\hline 7 & $43.5(\mathrm{~A})$ & $43.5(\mathrm{~A})$ \\
\hline 13 & $127.5(\mathrm{E})$ & 127.5(E) \\
\hline $3^{1}$ & 113.8(A) & 113.8(A) \\
\hline $3^{2}$ & 111.9(E) & $112.0(\mathrm{E})$ \\
\hline 10 & 108.3(E) & 108.2(E) \\
\hline 15 & $102.1(\mathrm{E})$ & 101.9(E), 100.4(E) \\
\hline 5 & 96.8(E), 95.9(E) & $96.4(\mathrm{E})$ \\
\hline 20 & $91.5(\mathrm{E})$ & 92.4(E), 90.9(E) \\
\hline 17 & $52.4(\mathrm{~A})$ & $52.4(\mathrm{~A})$ \\
\hline $17^{1}$ & $29.0(\mathrm{~A})$ & - \\
\hline 18 & 47.1(A) & - \\
\hline
\end{tabular}

Absorptive signals are assigned to the donor dimer, emissive signals are assigned to the acceptor cofactors (Surendran Thamarath et al. 2012a)

Labels (A) indicate absorptive and (E) emissive signals, respectively

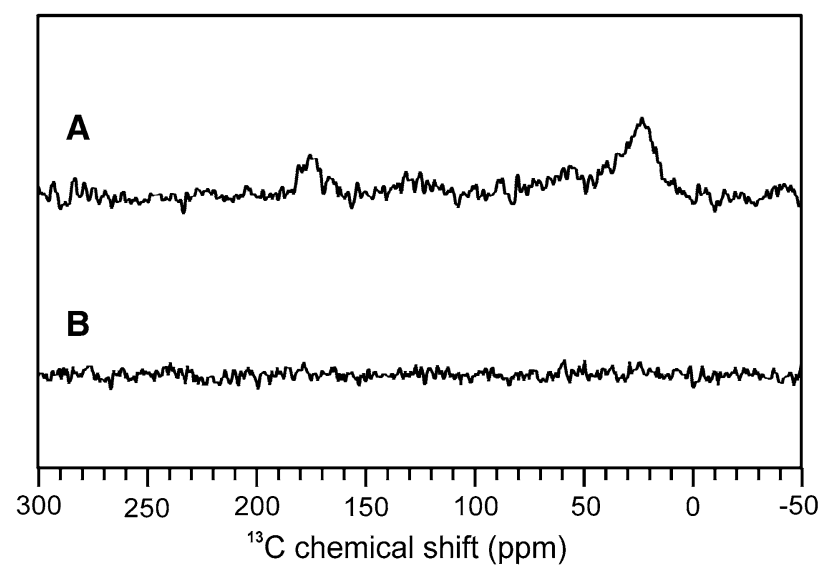

Fig. $6{ }^{13} \mathrm{C}$ MAS NMR spectra of Grünstoff at $A 4.7$ and $B 2.4 \mathrm{~T}$. Spectra are obtained from whole cells in the dark

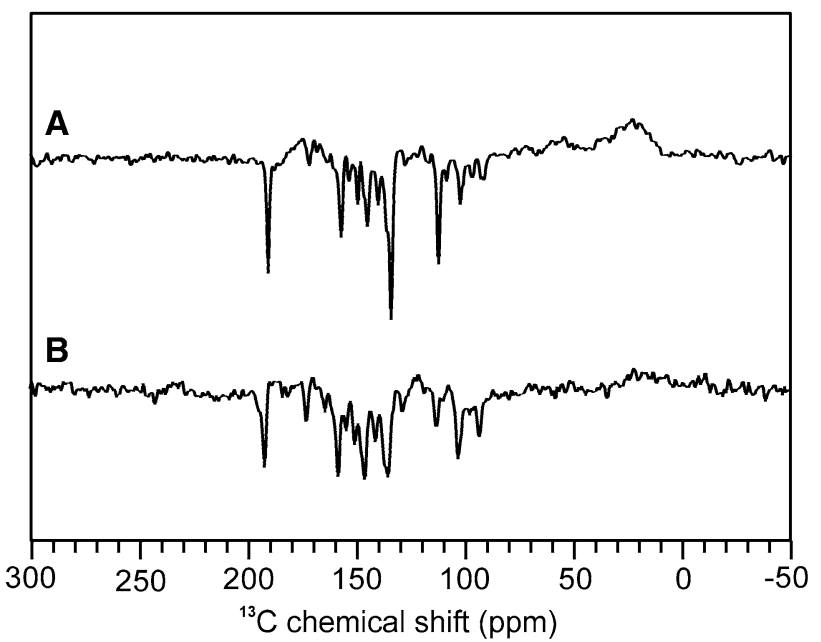

Fig. $7{ }^{13} \mathrm{C}$ photo-CIDNP MAS NMR spectra of Grünstoff at $A 4.7$ and $B 2.4 \mathrm{~T}$. Spectra are obtained from whole cells under illumination with continuous white light

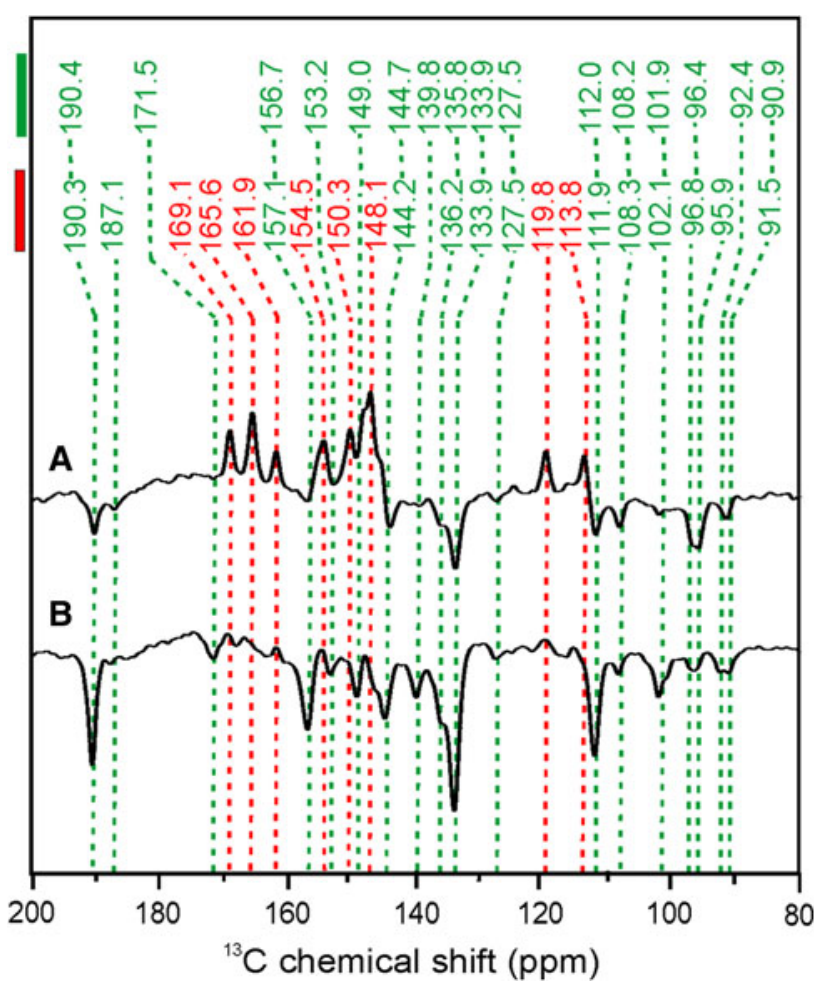

Fig. 8 Aromatic part of the ${ }^{13} \mathrm{C}$ photo-CIDNP MAS NMR spectra of anaerobically (Braunstoff) $(A)$ and aerobically (Grünstoff) $(B)$ treated cells of $\mathrm{Hb}$. mobilis. Chemical shift values denoted parallel to the red bar belong to Braunstoff and the assignments parallel to the green bar belong to Grünstoff. Red lines are the absorptive peaks and green lines are emissive peaks

and DD (enhanced absorptive). That would imply that TSM and $\mathrm{DD}$ are active in the entire observed field range. In this case, the DR would be active at $9.4 \mathrm{~T}$ and below. Peaking of the DR enhancement at a field lower than that for the peak 
of the combined TSM and DD enhancement has also been observed for RCs of $R b$. sphaeroides (Surendran Thamarath et al. 2012b).

From Grünstoff, the experimental data are presently less comprehensively but also here the modification of emissive signals can be explained by an alteration of the competition between TSM and DD. The DR is weak, an assumption possible because in this form the molecular triplet is born on an accessory cofactor and moves on a microsecond timescale to the donor (Surendran Thamarath et al. 2012a).

Comparison to other RCs and radical-pair lifetime

According to our interpretation, the spectra of Braunstoff show TSM and DD contributions over the entire field range, and are similar to PS I (Roy et al. 2007), while in PS II almost no enhancement effect is observed at 17.6 T (Roy et al. 2007). Since TSM and DD strongly depend on distance and orientation of cofactors that form the radical pair, the present data confirm the structurally close relation between RCs of heliobacteria and PS I. The spectral enhancement window is very broad as in RCs of $R b$. sphaeroides and PS I, providing evidence for a similar short timescale for the radical pair lifetime which is known to be in the range of some tens of nanoseconds.

The short lifetime of the radical pair in the photosynthetic primary process is related to two aspects of the functional mechanism of RCs: (i) The short time scale allows for high quantum yield of the light-induced electron transfer along highly optimized efficient reaction coordinates, and (ii) the general detectability of the solid-state photo-CIDNP effect in natural RCs due to lifetime broadening. Hence, the observability of the effect might indeed provide a measure for efficiency, and therefore a route to optimize artificial photosynthesis.

Acknowledgments The authors thank to A.H.M de Wit for helping in culturing the bacteria. Helpful discussions with Prof. G. Jeschke and G.J. Janssen are acknowledged. F. Lefeber, K. Erkelens and Bryan Ferlez are gratefully acknowledged. Generous financial support of NWO $(818.02 .019,713.012 .001)$ is acknowledged. JHG is supported by the Division of Chemical Sciences, Geosciences, and Biosciences, Office of Basic Energy Sciences of the U.S. Department of Energy, via Grant DE-FG02-08ER15989.

\section{References}

Bennett AE, Rienstra CM, Auger M, Lakshmi KV, Griffin RG (1995) Heteronuclear decoupling in rotating solids. J Chem Phys 103:6951-6958

Brockmann H, Lipinski A (1983) Bacteriochlorophyll $g$ a new bacteriochlorophyll from Heliobacterium chlorum. Arch Microbiol 136:17-19

Closs GL (1975) Overhauser mechanism of chemically-induced nuclear-polarization as suggested by Adrian. Chem Phys Lett $32: 277-278$
Closs GL (1977) In: Muus LT, Atkins PW, McLauchlan KA, Pedersen JB, Chemically induced magnetic polarization, NATO Advanced study institutes series, Reidel, Dordrecht, pp. 225-256

Daviso E, Jeschke G, Matysik J (2008) Photochemically induced dynamic nuclear polarization (photo-CIDNP) magic-angle spinning NMR. In: Aartsma T, Matysik J (eds) Biophysical Techniques in Photosynthesis II. Springer, Dordrecht, pp 385-399

Daviso E, Alia A, Prakash S, Diller A, Gast P, Lugtenburg J, Matysik J, Jeschke G (2009) Electron-nuclear spin dynamics in a bacterial photosynthetic reaction center. J Phys Chem C 113:10269-10278

Daviso E, Janssen GJ, Alia A, Jeschke G, Matysik J, Tessari M (2011) A 10000 -fold nuclear hyperpolarization of a membrane protein in the liquid phase via solid-state mechanism. J Am Chem Soc 133:16754-16757

Fischer MR, de Groot HJM, Raap J, Winkel C, Hoff AJ, Lugtenburg J (1992) C-13 magic-angle spinning NMR study of the lightinduced and temperature-dependent changes in Rhodobacter sphaeroides R26 reaction centers enriched in [4'-C-13]tyrosine. Biochemistry 31:11038-11049

Gest H (1994) Discovery of the heliobacteria. Photosynth Res 41:17-21

Golbeck J (2007) The heliobacterial reaction center. Photosynth Res 91:139-140

Goldstein RA, Boxer SG (1987) Effects of nuclear-spin polarization on reaction dynamics in photosynthetic bacterial Reaction Centers. Biophys J 51:937-946

Janssen GJ, Daviso E, van Son M, de Groot HJM, Alia A, Matysik J (2010) Observation of the solid-state photo-CIDNP effect in entire cells of cyanobacteria Synechocystis. Photosynth Res 104:275-282

Jeschke G (1997) Electron-electron-nuclear three-spin mixing in spincorrelated radical pairs. J Chem Phys 106:10072-10086

Jeschke G, Matysik J (2003) A reassessment of the origin of photochemically induced dynamic nuclear polarization effects in solids. Chem Phys 294:239-255

Jeschke G, Anger BC, Bode BE, Matysik J (2011) Theory of solidstate photo-CIDNP in the earth's magnetic field. J Phys Chem A 115:9919-9928

Kobayashi M, Vandemeent EJ, Erkelens C, Amesz J, Ikegami I, Watanabe T (1991) Bacteriochlorophyll g epimer as a possible reaction center component of heliobacteria. Biochim Biophys Acta 1057:89-96

Matysik J, Alia, Hollander JG, Egorova-Zachernyuk T, Gast P, de Groot HJM (2000) A set-up to study photochemically induced dynamic nuclear polarization in photosynthetic reaction centres by solid-state NMR. Indian J Biochem Biophys 37:418-423

Matysik J, Schulten E, Alia, Gast P, Raap J, Lugtenburg J, Hoff AJ, de Groot HJM (2001) Photo-CIDNP C-13 magic angle spinning NMR on bacterial reaction centres: exploring the electronic structure of the special pair and its surroundings. Biol Chem 382:1271-1276

Matysik J, Diller A, Roy E, Alia A (2009) The solid-state photoCIDNP effect. Photosynth Res 102:427-435

McDermott A, Zysmilich MG, Polenova T (1998) Solid state NMR studies of photoinduced polarization in photosynthetic reaction centers: mechanism and simulations. Solid State Nucl Magn Reson 11:21-47

Nabedryk E, Leibl W, Breton J (1996) FTIR spectroscopy of primary donor photooxidation in photosystem I, Heliobacillus mobilis, and Chlorobium limicola. Comparison with purple bacteria. Photosynth Res 48:301-308

Polenova T, McDermott AE (1999) A coherent mixing mechanism explains the photoinduced nuclear polarization in photosynthetic reaction centers. J Phys Chem B 103:535-548 
Prakash S, Alia, Gast P, de Groot HJM, Jeschke G, Matysik J (2005) Magnetic field dependence of photo-CIDNP MAS NMR on photosynthetic reaction centers of Rhodobacter sphaeroides WT. J Am Chem Soc 127:14290-14298

Prakash S, Alia, Gast P, de Groot HJM, Matysik J, Jeschke G (2006) Photo-CIDNP MAS NMR in intact cells of Rhodobacter sphaeroides R26: Molecular and atomic resolution at nanomolar concentration. J Am Chem Soc 128:12794-12799

Prince RC, Gest H, Blankenship RE (1985) Thermodynamic properties of the photochemical-reaction center of Heliobacterium chlorum. Biochim Biophys Acta 810:377-384

Roy E, Diller A, Alia, Gast P, van Gorkom HJ, de Groot HJM, Jeschke G, Matysik J (2007) Magnetic field dependence of ${ }^{13} \mathrm{C}$ photo-CIDNP MAS NMR in plant photosystems I and II. Appl Magn Reson 31:193-204

Roy E, Rohmer T, Gast P, Jeschke G, Alia A, Matysik J (2008a) Characterization of the primary radical pair in reaction centers of Heliobacillus mobilis by ${ }^{13} \mathrm{C}$ photo-CIDNP MAS NMR. Biochemistry 47:4629-4635

Roy E, Alia A, Gast P, van Gorkom HJ, Jeschke G, Matysik J (2008)

${ }^{13} \mathrm{C}$ photo-CIDNP MAS NMR on the reaction center of the green sulphur bacterium at two different magnetic fields In: Allen $\mathrm{J}$, Gantt E, Golbeck J, Osmond B (eds). Energy from the sun. Springer, Dordrecht, pp 173-176

Stevenson AK, Kimble LK, Woese CR, Madigan MT (1997) Characterization of new phototrophic heliobacteria and their habitats. Photosynth Res 53:1-11
Surendran Thamarath S, Heberle J, Hore PJ, Kottke T, Matysik J (2010) Solid-state photo-CIDNP effect observed in Phototropin LOV1-C57S by ${ }^{13} \mathrm{C}$ magic-angle spinning NMR spectroscopy. J Am Chem Soc 132:15542-15543

Surendran Thamarath S, Alia A, Daviso E, Mance D, Golbeck JH, Matysik J (2012a) Whole-cell NMR characterization of two photochemically active states of the photosynthetic reaction center in heliobacteria. Biochemistry 51:5763-5773

Surendran Thamarath S, Bode BE, Prakash S, Sai Sankar Gupta KB, Alia A, Jeschke G, Matysik J (2012b) Electron spin density distribution in the special pair triplet of Rhodobacter sphaeroides R26 revealed by magnetic field dependence of the solidstate photo-CIDNP effect. J Am Chem Soc 134:5921-5930

van de Meent EJ, Kleinherenbrink FAM, Amesz J (1990) Purification and properties of an antenna-reaction center complex from Heliobacteria. Biochim Biophys Acta 1015:223-230

Vandemeent EJ, Kobayashi M, Erkelens C, Vanveelen PA, Amesz J, Watanabe T (1991) Identification of $8^{1}$-hydroxychlorophyll $a$ as a functional reaction center pigment in heliobacteria. Biochim Biophys Acta 1058:356-362

Vassiliev IR, Antonkine ML, Golbeck JH (2001) Iron-sulfur clusters in type I reaction centers. Biochim Biophys Acta 1507:139-160

Zysmilich MG, McDermott A (1994) Photochemically induced dynamic nuclear-polarization in the solid-State N-15 spectra of reaction centers from photosynthetic bacteria Rhodobacter sphaeroides R26. J Am Chem Soc 116:8362-8363 